\title{
Envelhecimento acelerado para avaliação do potencial fisiológico de se- mentes de melão
}

\author{
Salvador B Torres; Fabrícia N de Oliveira; Andreya K de Oliveira; Clarisse P Benedito; Jean C \\ Marinho \\ UFERSA, Programa Pós-Graduação em Fitotecnia, C Postal 137, 59.625-900 Mossoró-RN; sbtorres@ufersa.edu.br
}

\section{RESUMO}

O teste de envelhecimento acelerado tem potencial para ser utilizado no controle de qualidade de sementes de hortaliças. No entanto, há fatores que podem afetar os resultados, sendo necessários ajustes na metodologia para obtenção de resultados consistentes. Esta pesquisa foi conduzida com o objetivo principal de avaliar procedimentos para a condução do teste de envelhecimento acelerado para avaliação do potencial fisiológico de sementes de melão, incluindo o uso de solução saturada de $\mathrm{NaCl}$ em substituição à água. Sementes de dois híbridos de melão (Goldex e Vereda), cada um representados por cinco lotes, foram submetidos aos testes de germinação, primeira contagem de germinação, condutividade elétrica (16 e 24 horas de embebição), emergência de plântulas em casa de vegetação e envelhecimento acelerado. Neste, foram empregados dois procedimentos: tradicional (água) e solução saturada $\left(40 \mathrm{~g} 100 \mathrm{~mL}^{-1}\right) \mathrm{de} \mathrm{NaCl}$, a $41^{\circ} \mathrm{C}$, por períodos de 24,48 e 72 horas. Foi utilizado o delineamento inteiramente casualizado, com quatro repetições e análises isoladas para cada teste. Os testes de primeira contagem de germinação e emergência de plântulas foram eficientes na identificação dos lotes de sementes de qualidade inferior. Já o teste de condutividade elétrica não se constituiu em opção eficiente para avaliação do vigor das sementes. O teste de envelhecimento acelerado (procedimento tradicional e com solução salina saturada), utilizando o período de 72 horas, a $41^{\circ} \mathrm{C}$, mostra-se adequado para avaliação do potencial fisiológico de sementes de melão. Observou-se ainda que, em comparação com o uso de água, a utilização de solução salina no teste de envelhecimento acelerado inibiu sensivelmente o crescimento e desenvolvimento de fungos.

\section{ABSTRACT}

Accelerated aging test to evaluate the physiological potential of melon seeds

The accelerated aging test can be useful for quality control of vegetable seeds. However, there are factors which can affect the results, so it is necessary to adjust the methodology to obtain consistency. This study was carried out with the main objective of evaluating procedures for performing accelerated aging test to estimate the physiological potential of melon seeds, including the use of saturated solution of $\mathrm{NaCl}$ of water. Seeds of two melon hybrids (Goldex and Vereda), each one represented by five lots, were submitted to germination tests, first germination counting, electrical conductivity (16 and 24 hours of imbibition), plant emergence in greenhouse and accelerated aging. In the last, two procedures were used: traditional (water) and saturated solution $\left(40 \mathrm{~g} 100 \mathrm{~mL}^{-1}\right)$ of $\mathrm{NaCl}$ at $41^{\circ} \mathrm{C}$ for 24,48 and 72 hours. A completely random design was used with four replications and single analysis for each test. The tests of first germination counting and plant emergence were efficient to identify seed lots of inferior quality. But the electrical conductivity test was not efficient for evaluation of seed vigor. The accelerated aging test (traditional procedure and with saline saturated solution), using the period of 72 hours at $42^{\circ} \mathrm{C}$, shows to be adequate for evaluating the physiological potential of melon seeds. In comparison with the use of water, the utilization of saline solution in the accelerated aging test inhibited significantly growth and development of fungi.

Keywords: Cucumis melo, vigor, germination.

Palavras-chave: Cucumis melo, vigor, germinação.

(Recebido para publicação em 6 de novembro de 2007; aceito em 15 de dezembro de 2008)

(Received in November 6, 2007; accepted in December 15, 2008)

$\mathrm{D}$ entre as espécies hortícolas, o melão (Cucumis melo L.), vem sendo produzido em volumes significativos, principalmente na região Nordeste do Brasil. Constitui-se na segunda fruta mais exportada na última safra (2005/ 2006), com o crescimento de $44 \%$ em volume e $26 \%$ em valor. Atualmente, as exportações brasileiras de melão chegam a US\$ 58 milhões, representando 17,4\% do total das exportações brasileiras de frutas frescas (CNPq, 2007).

As sementes dos materiais híbridos, por apresentarem alto valor comercial, merecem atenção especial quanto ao potencial fisiológico. Entretanto, para esta espécie, ainda são poucas as pesquisas direcionadas à comparação de testes de vigor, sobretudo em nossas condições.

A avaliação do potencial fisiológico de sementes é componente fundamental de programas de controle de qualidade, pois constitui referência para adoção de práticas de manejo destinadas à garantia de nível satisfatório de desempenho. O teste de germinação, conduzido em condições favoráveis de substrato, umidade e temperatura, geralmente superestima o potencial fisio- lógico de lotes de sementes sendo, portanto, cada vez maior a necessidade do uso e aprimoramento de testes de vigor para avaliar o potencial fisiológico de sementes.

O envelhecimento acelerado como teste de vigor tem sido um dos mais utilizados, com possibilidade de aplicação para sementes de diversas culturas (McDonald, 1995). Nesse teste, as sementes são submetidas à alta temperatura e umidade relativa elevada por período relativamente curto, sendo, em seguida, colocadas para germinar. Lotes de sementes de alto vigor manterão 
sua viabilidade quando submetidos a essas condições, enquanto os de baixo vigor terão sua viabilidade reduzida (AOSA, 1983).

Embora o teste de envelhecimento já tenha caminhado suficientemente em direção à padronização, para muitas espécies, ainda há vários estudos em andamento com o objetivo de aprimorar sua metodologia (Marcos Filho, 1999a). A padronização de um teste envolve a identificação de todas as variáveis que influenciam os resultados e a incorporação de práticas adequadas de controle. No teste de envelhecimento acelerado as variáveis básicas são temperatura, umidade relativa do ar e período de exposição das sementes ao mesmo. Dentre estas, a interação temperatura/período de exposição é uma das mais estudadas. Na literatura pesquisada alguns autores dedicaram-se ao estudo dessa interação, indicando, para sementes de melancia, $41^{\circ} \mathrm{C} / 48 \mathrm{~h}$ (Bhering et al., 2001); cenoura, $41^{\circ} \mathrm{C} / 48 \mathrm{~h}$ (Rodo et al., 2001); melão, $41^{\circ} \mathrm{C} / 72$ h ou 96 h (Torres \& Marcos Filho, 2003); pepino, $41^{\circ} \mathrm{C} / 72 \mathrm{~h}$ (Abdo et al., 2005); lentilha, $41^{\circ} \mathrm{C} / 48 \mathrm{~h}$ (Freitas \& Nascimento, 2006); rabanete, $41^{\circ} \mathrm{C} / 48$ ou $72 \mathrm{~h}$ (Ávila et al., 2006); ervilha, $41^{\circ} \mathrm{C} / 48 \mathrm{~h}$ (Nascimento et al., 2007).

Para as espécies de sementes pequenas, como as de hortaliças, o teste de envelhecimento acelerado pode apresentar certas limitações, como a desuniformidade de absorção de água entre amostras, o que pode resultar em deterioração diferenciada, comprometendo os resultados pós-envelhecimento. Portanto, para minimizar esse problema, Jianhua \& McDonald (1996) propuseram, durante a condução do teste, a substituição de água por soluções saturadas de sais. Com esse procedimento, há redução da umidade relativa do ambiente, retardando assim a absorção de água pelas sementes, conforme verificaram Torres \& Marcos Filho (2003, 2005), também com sementes de melão. Esse método possibilita ainda redução de infecção por microorganismos durante o teste, o que pode mascarar os resultados. Esse fato foi observado em sementes de pepino (Abdo et al., 2005), rabanete (Ávila et al., 2006), ervilha (Nascimento et al., 2007) e brássicas (couve, couve-brócolis e repolho) (Costa et al., 2008).
O presente trabalho teve por objetivo estabelecer metodologia para condução do teste de envelhecimento acelerado para avaliação do potencial fisiológico de sementes de melão dos híbridos Goldex e Vereda.

\section{MATERIAL E MÉTODOS}

A pesquisa foi conduzida em laboratório de análise de sementes da UFERSA, em Mossoró, de dezembro/06 a março/07. Foram utilizadas sementes de dois híbridos de melão (Vereda e Goldex), cada um representado por cinco lotes, provenientes de produtores da região do pólo agrícola Assú-BaraúnaMossoró (RN). Após a recepção, as sementes foram homogeneizadas em divisor tipo Gamet, e acondicionadas em saco de papel kraft e armazenadas em condições controladas $\left(18-20^{\circ} \mathrm{C}\right.$ e $50 \%$ de umidade relativa do ar), permanecendo nessas condições até o final da fase experimental. As avaliações da qualidade das sementes foram realizadas pelos testes descritos a seguir. Teor de água: realizado em estufa a $105 \pm 3^{\circ} \mathrm{C} / 24 \mathrm{~h}$ (Brasil, 1992), utilizando-se duas amostras com 4,0 g de sementes cada, para cada lote. Os resultados foram expressos em porcentagem (base úmida); Germinação: utilizaram-se quatro subamostras de 50 sementes por lote, distribuídas sobre duas folhas de papel toalha tipo germitest, umedecidas com água destilada em quantidade equivalente a duas vezes e meia a sua massa. Em seguida, as sementes foram cobertas com mais uma folha de papel, confeccionando-se os rolos, que foram levados ao germinador, regulado a $25^{\circ} \mathrm{C}$. As avaliações foram realizadas aos quatro e oito dias após a semeadura, segundo os critérios estabelecidos pelas Regras para Análise de Sementes (Brasil, 1992); os resultados foram expressos em porcentagem de plântulas normais; Primeira contagem de germinação: realizada conjuntamente com o teste de germinação, com avaliação da porcentagem de plântulas normais no quarto dia (Brasil, 1992); Condutividade elétrica: baseado em resultados preliminares, este teste conduzido pelo método de massa, com quatro subamostras de 25 sementes fisicamente puras, que após pesadas fo- ram colocadas para embeber em copos de plástico $(200 \mathrm{~mL})$, contendo $50 \mathrm{~mL}$ de água destilada a $25^{\circ} \mathrm{C}$ durante 16 e 24 horas. Após o condicionamento, a condutividade elétrica da solução foi medida por meio de leituras em condutivímetro Digimed, modelo 31, e os resultados expressos em $\mu \mathrm{S} \mathrm{cm} \mathrm{cm}^{-1} \mathrm{~g}^{-1}$. A leitura foi realizada logo após a retirada do material da incubadora, de modo gradativo, agitando-se cuidadosamente cada recipiente, com o intuito de uniformizar os eletrólitos lixiviados na solução (Vieira \& Krzyzanowski, 1999); Emergência de plântulas em casa de vegetação: foram utilizadas quatro subamostras de 50 sementes por lote, distribuídas em bandejas multicelulares de "isopor", com células individuais, contendo substrato comercial, composto por casca de pinus, fibra de coco, vermiculita e fertilizante (informações fornecidas pelo fabricante). As bandejas foram mantidas em temperatura ambiente $\left(27\right.$ a $\left.30^{\circ} \mathrm{C}\right)$ e irrigação periódica. Após as contagens, efetuadas aos doze dias após a semeadura, determinou-se a porcentagem de emergência de plântulas (Nakagawa, 1994); Envelhecimento acelerado (procedimento tradicional): utilizou-se a metodologia proposta pela AOSA (1983) e descrita por Marcos Filho (1999b). Foram distribuídas quatro gramas de sementes sobre tela de alumínio, fixada em caixa plástica de germinação $(11 \times 11 \times 3,5 \mathrm{~cm})$, contendo no fundo $40 \mathrm{~mL}$ de água destilada. As caixas contendo as sementes foram tampadas e permaneceram no interior da câmara a $41^{\circ} \mathrm{C}$, por períodos de 24,48 e 72 horas. Em seguida, as sementes foram colocadas para germinar conforme metodologia descrita para o teste de germinação. A avaliação foi realizada aos quatro dias após a semeadura e, os resultados, expressos em porcentagem. Para fins de monitoramento do teste, foi determinado, também, o teor de água das sementes antes e após o envelhecimento das sementes. Envelhecimento acelerado (solução saturada de sal): conduzido de maneira similar à descrita para o teste tradicional, com exceção de serem adicionados ao fundo de cada caixa plástica (compartimento individual), $40 \mathrm{~mL}$ de solução saturada de $\mathrm{NaCl}$, em substituição à água. Essa solução foi obtida pela 
Tabela 1. Germinação, primeira contagem, condutividade elétrica (CE) (16h e 24h de embebição) e emergência de plântulas em casa de vegetação de sementes de dez lotes de melão, híbridos Goldex e Vereda (germination, first counting, electrical conductivity (CE) (16h and 24h of soaking) and seedling emergence in the green house of melon seeds, hybrids Goldex and Vereda). Mossoró, UFERSA, 2007.

\begin{tabular}{|c|c|c|c|c|c|c|}
\hline \multirow{2}{*}{ Híbrido } & \multirow{2}{*}{ Lote } & \multirow{2}{*}{$\begin{array}{c}\text { Germinação } \\
(\%)\end{array}$} & \multirow{2}{*}{$\begin{array}{c}\text { Primeira } \\
\text { contagem } \\
(\%)\end{array}$} & \multicolumn{2}{|c|}{$\begin{array}{c}\text { Condutividade elétrica } \\
\left(\mu \mathrm{S} \mathrm{cm} \mathrm{cm}^{-1} \mathrm{~g}^{-1}\right)\end{array}$} & \multirow{2}{*}{$\begin{array}{c}\text { Emergên-- } \\
\text { cia de } \\
\text { plântulas } \\
(\%)\end{array}$} \\
\hline & & & & $16 \mathrm{~h}$ & $24 \mathrm{~h}$ & \\
\hline \multirow[t]{6}{*}{ Goldex } & 1 & $92 a$ & 80 & $75 a$ & $97 \mathrm{a}$ & $86 \mathrm{~b}$ \\
\hline & 2 & 96 a & 90 & $86 \mathrm{~b}$ & $100 \mathrm{~b}$ & 94 a \\
\hline & 3 & 98 a & 89 & $100 \mathrm{c}$ & $124 \mathrm{c}$ & $94 \mathrm{a}$ \\
\hline & 4 & 96 a & 92 & $102 \mathrm{c}$ & $130 \mathrm{c}$ & 96 a \\
\hline & 5 & 96 a & 90 & $74 \mathrm{a}$ & 99 a & $94 \mathrm{a}$ \\
\hline & CVs (\%) & 3,4 & 4,6 & 4,8 & 5,3 & 6,2 \\
\hline \multirow[t]{6}{*}{ Vereda } & 1 & $98 \mathrm{a}$ & $96 \quad a$ & $78 a$ & $96 \mathrm{a}$ & $94 \mathrm{a}$ \\
\hline & 2 & 96 a & 90 & $100 \mathrm{~b}$ & $111 \mathrm{~b}$ & $87 \mathrm{~b}$ \\
\hline & 3 & 94 a & $a b$ & $78 a$ & 99 a & $91 a b$ \\
\hline & 4 & $92 \mathrm{a}$ & 89 & $125 c$ & $143 c$ & $88 \mathrm{~b}$ \\
\hline & 5 & $86 \mathrm{~b}$ & $74 \mathrm{c}$ & $75 a$ & $95 \mathrm{a}$ & $81 \mathrm{c}$ \\
\hline & CVs (\%) & 4,2 & 5,7 & 6,1 & 5,9 & 7,7 \\
\hline
\end{tabular}

Médias seguidas pela mesma letra na coluna não diferem entre si, pelo teste de Tukey, $\mathrm{p} \leq 0,05$, para cada híbrido (means followed by the same letter in the column did not differ from each other, Tukey's test, $\mathrm{p} \leq 0,05)$.

proporção $40 \mathrm{~g} \mathrm{de} \mathrm{NaCl}$ (p.a.) $100 \mathrm{~mL}^{-1}$ de água, estabelecendo, com isso, ambiente com umidade relativa do ar de $76 \%$ (Jianhua \& McDonald, 1996).

O experimento foi conduzido no delineamento inteiramente casualizado, com quatro repetições. Os dados foram submetidos aos testes de normalidade e de homogeneidade de variância, que indicaram a não necessidade de transformação. Em seguida, foram submetidas à análise de variância, sendo a comparação de médias efetuada pelo teste de Tukey, a 5\% de probabilidade.

\section{RESULTADOS E DISCUSSÃO}

Verificou-se pelo teste de germinação que os lotes do híbrido Goldex não diferiram entre si. Com relação ao híbrido Vereda, o lote 5 foi considerado como de qualidade inferior, enquanto os lotes 1, 2, 3, e 4 foram agrupados num mesmo nível, não diferindo entre si (Tabela 1).

Todos os lotes apresentaram germinação superior à mínima $(80 \%)$ estabelecida para comercialização de sementes de melão (CESM/RS, 1998). É importante e coerente a comparação de lotes de sementes com germinação semelhante (Marcos Filho, 1999a) e, de acordo com Powell (1986), preferencial- mente situados na Fase I da curva de perda de viabilidade, pois, ao atingir a Fase II, mesmo o teste de germinação (conduzido sob condições favoráveis) é capaz de detectar diferenças no potencial fisiológico das amostras avaliadas. Essa autora considera que a posição de cada lote dentro da Fase I determina seu nível de vigor. Neste estudo, todos os lotes apresentaram germinação variando entre 86 e $98 \%$, estando situados, portanto, na Fase I da curva de perda de viabilidade da semente, caracterizada por ser relativamente longa e com poucas sementes mortas.

O teste de primeira contagem de germinação (Tabela 1), mostrou-se mais sensível que o teste de germinação, principalmente para os lotes do híbrido Vereda, que classificou o lote 1 como de melhor potencial fisiológico, embora não diferindo estatisticamente do lote 3 ; o lote 5 foi identificado como de pior qualidade. Com relação aos lotes do híbrido Goldex, esses apresentaram resultados semelhantes no teste de germinação, diferindo apenas para o lote 1 que apresentou menor valor para a primeira contagem.

Embora a primeira contagem de germinação possa ser considerada um indicativo de vigor, sabe-se que a redu- ção da velocidade da germinação não está ente os primeiros eventos do processo de deterioração de sementes (Delouche \& Baskin, 1973), justificando a menor eficiência deste teste em detectar pequenas diferenças de vigor e o conseqüente agrupamento dos lotes. Por outro lado, Bhering et al. (2000) trabalhando com sementes de pepino, verificaram que a primeira contagem do teste de germinação pode ser utilizado rotineiramente para se obterem informações preliminares sobre o vigor dos lotes de sementes desta espécie.

Analisando os resultados do teste de condutividade elétrica (Tabela 1), verificou-se para ambos os híbridos que os períodos de embebição (16 e 24 horas) mostraram concordância quanto à ordenação dos lotes e a possibilidade de redução no período de condicionamento das sementes de melão, sem que haja prejuízo de confiabilidade dos resultados. Esta possibilidade de redução do período de embebição em sementes de hortaliças foi também verificada por Torres et al. (1998), com maxixe; Dias et al. (1998), com quiabo; Sá (1999), com tomate e Abdo et al. (2005), com pepino.

Neste teste os lotes 1 e 5 do híbrido Goldex foram indicados como os mais vigorosos, enquanto os lotes 3 e 4 como de qualidade inferior; o lote 2 apresentou resultado intermediário. Portanto, observa-se que a condutividade elétrica não forneceu informações compatíveis com os demais testes empregados, exceto para o lote 5 que manteve a superioridade. Para o híbrido Vereda, os lotes 1,3 e 5 foram considerados como de melhor potencial fisiológico e o lote 4 como de pior desempenho; o lote 2 apresentou comportamento intermediário. Para este híbrido, os resultados de condutividade elétrica também não foram totalmente coerentes com os obtidos nos testes de germinação e primeira contagem de germinação que, para estes testes, o lote 5 é tido como de pior qualidade.

O teste de condutividade tem sido reconhecido como eficiente para a avaliação do vigor de hortaliças, conforme demonstraram Oliveira \& Novembre (2005), para pimentão; Abdo et al. (2005), para pepino; Dias et al (2006), para cebola e Vieira \& Dutra (2006), para abó- 
bora. No entanto, o teste não tem sido considerado eficiente para melão (Torres \& Marcos Filho, 2005) e tomate (Novembre et al., 1995). Nestas espécies, provavelmente a presença da membrana semi-permeável, de origem nucelar, permite a entrada de água mas não a difusão de certos eletrólitos para o exterior. Fato também verificado nesta pesquisa, onde o teste de condutividade elétrica mostrou-se menos adequado para avaliação do vigor de sementes de melão, uma vez que seus resultados se mostraram contraditórios aos obtidos nos outros testes, em maior ou menor intensidade, dependendo do híbrido avaliado. Entretanto, a continuidade da pesquisa provavelmente fornecerá subsídios para o esclarecimento dos motivos que determinam a obtenção de resultados imprevisíveis com esse teste.

$\mathrm{O}$ teste de emergência de plântulas em casa de vegetação (Tabela 1) classificou os lotes de melhor e pior desempenho, de maneira semelhante à verificada no teste de primeira contagem de germinação. Segundo Marcos Filho (1999a), o teste de emergência de plântulas constitui parâmetro indicador da eficiência dos testes para avaliação do potencial fisiológico de lotes de sementes. Portanto, essa eficiência em distinguir, com segurança, os lotes de baixo e alto vigor foi mais evidente para os lotes do híbrido Vereda, enquanto que os do híbrido Goldex, apresentaram qualidade relativamente uniforme, identificando-se apenas o lote 1 como de menor potencial fisiológico. Assim, enfatiza-se a importância do uso de mais de um teste para determinar o vigor dos lotes de sementes (Marcos Filho, 1998), devido à influência dos métodos adotados e uso de situações específicas de estresse para estimar o comportamento relativo dos lotes em campo (Carvalho \& Nakagawa, 2000).

O teor de água inicial das sementes foi semelhante para os dez lotes (Tabela 2). Este fato é importante para a execução dos testes, considerando-se que a uniformização do teor de água das sementes é imprescindível para a padronização dos procedimentos e obtenção de resultados consistentes (Marcos Filho, 1999b), pois, dentro de certos limites, as sementes mais úmidas são mais afetadas pelas condições do envelhecimen-

Tabela 2. Teor de água (TA) obtido antes e após os períodos de envelhecimento, a $41^{\circ} \mathrm{C}$, em sementes de melão, híbridos Goldex e Vereda (seed moisture content obtained before and after the seed aging, at $41^{\circ} \mathrm{C}$, in melon seeds, hybrids Godex and Vereda). Mossoró, UFERSA, 2007.

\begin{tabular}{|c|c|c|c|c|c|c|c|c|}
\hline \multirow{3}{*}{ Híbrido } & \multirow{3}{*}{ Lote } & \multicolumn{7}{|c|}{ Teor de água (\%) } \\
\hline & & \multirow{2}{*}{ Inicial } & \multicolumn{3}{|c|}{ Tradicional } & \multicolumn{3}{|c|}{ Solução saturada } \\
\hline & & & $24 h$ & $48 h$ & $72 \mathrm{~h}$ & $24 \mathrm{~h}$ & $48 h$ & $72 \mathrm{~h}$ \\
\hline \multirow[t]{5}{*}{ Goldex } & 1 & 7,2 & 25,4 & 26,8 & 27,3 & 8,8 & 9,6 & 9,6 \\
\hline & 2 & 7,0 & 24,9 & 27,0 & 28,0 & 8,6 & 9,2 & 9,4 \\
\hline & 3 & 7,2 & 24,4 & 27,0 & 27,6 & 7,8 & 10,0 & 10,2 \\
\hline & 4 & 7,5 & 25,6 & 27,2 & 27,8 & 9,2 & 9,8 & 9,6 \\
\hline & 5 & 6,9 & 24,6 & 25,3 & 26,6 & 8,4 & 9,6 & 9,4 \\
\hline \multirow[t]{5}{*}{ Vereda } & 1 & 7,0 & 25,4 & 26,8 & 27,2 & 8,8 & 9,7 & 9,6 \\
\hline & 2 & 6,5 & 26,2 & 28,0 & 29,1 & 8,6 & 9,8 & 10,0 \\
\hline & 3 & 7,0 & 25,4 & 27,0 & 28,4 & 8,6 & 9,6 & 10,0 \\
\hline & 4 & 7,4 & 26,1 & 28,2 & 28,8 & 9,2 & 10,1 & 10,2 \\
\hline & 5 & 7,1 & 25,6 & 26,6 & 27,2 & 9,3 & 10,2 & 10,1 \\
\hline
\end{tabular}

to acelerado, apresentando maior grau de deterioração.

As sementes de melão envelhecidas pelo método tradicional apresentaram acréscimos no teor de água com o aumento do período de envelhecimento. Verificou-se que esse incremento foi mais acentuado no primeiro período de envelhecimento, ou seja, após 24 horas (Tabela 2). A partir deste período, o teor de água das sementes continuou aumentando, porém em proporções inferiores, quando comparadas ao primeiro período de envelhecimento, mostrando, portanto, que as sementes secas, por apresentarem baixo potencial mátrico, absorvem água rapidamente quando colocadas em atmosfera úmida (Bewley \& Black, 1994). O teor de água das sementes expostas à solução saturada de $\mathrm{NaCl}$ apresentou valores menores e mais uniformes, após os períodos de envelhecimento em relação aos observados para as envelhecidas pelo procedimento tradicional (Tabela 2); isto indica que o uso de solução salina contribuiu para retardar a absorção de água pelas sementes durante o envelhecimento das mesmas.

Verificou-se que, de forma geral, ambos os procedimentos de envelhecimento (Tabela 3), proporcionaram a identificação dos lotes 1 e 5 como de menor potencial fisiológico, respectivamente para os híbridos Goldex e Vereda, havendo, nesse sentido concordância com os testes de primeira contagem de germinação e emergência das plântulas em casa de vegetação (Tabela 1).

Apesar da semelhança entre as informações proporcionadas para os hí- bridos Goldex e Vereda (Tabela 3), indicando os lotes de menor e maior qualidade, os períodos de 24 e 48 horas de envelhecimento, mostraram-se pouco eficientes na separação dos lotes em diferentes níveis de vigor. Utilizando o período de 48 horas, $\mathrm{a} 42^{\circ} \mathrm{C}$, para avaliação de sementes de melão, Cano-Ríos et al. (2000), também constataram que este período mostrou-se pouco sensível para estimar o potencial de armazenamento das sementes.

Neste estudo, constatou-se que o período de 72 horas, para ambos os procedimentos de envelhecimento, exibiu maior sensibilidade para identificação dos lotes em diferentes níveis de potencial fisiológico. Dessa forma, o híbrido Goldex apresentou os lotes 2, 3 e 4 como de melhor potencial fisiológico; o lote 1 como de pior qualidade e o 5 com qualidade intermediária. Esses resultados concordam em parte com os obtidos para os testes de primeira contagem de germinação e emergência de plântulas em casa de vegetação quando indicam a inferioridade do lote 1 (Tabela 1). Os resultados do híbrido Vereda indicam que os lotes 1 e 5 apresentaram melhor e pior qualidade, respectivamente; de forma geral, os lotes 2, 3 e 4 revelaram-se como de qualidade intermediária, havendo, nesse aspecto, concordância com os resultados dos testes de primeira contagem e emergência das plântulas (Tabela 1). Esse período de 72 horas de envelhecimento, tanto no procedimento tradicional quanto em solução saturada de $\mathrm{NaCl}$, corrobora com o encontrado por Torres 
Tabela 3. Vigor avaliado pelo teste de envelhecimento acelerado tradicional e com solução saturada de $\mathrm{NaCl}, \mathrm{a} 41^{\circ} \mathrm{C}$, em sementes de melão, híbridos Goldex e Vereda (vigor evaluated by the accelerated aging test and $\mathrm{NaCl}$ saturated solution, at $41^{\circ} \mathrm{C}$, in melon seeds, hybrids Goldex and Vereda). Mossoró, UFERSA, 2007.

\begin{tabular}{|c|c|c|c|c|c|c|c|}
\hline \multirow{3}{*}{ Híbrido } & \multirow{3}{*}{ Lote } & \multicolumn{6}{|c|}{ Vigor (\%) } \\
\hline & & \multicolumn{3}{|c|}{ Tradicional } & \multicolumn{3}{|c|}{ Solução saturada } \\
\hline & & $24 \mathrm{~h}$ & $48 \mathrm{~h}$ & $72 \mathrm{~h}$ & $24 \mathrm{~h}$ & $48 \mathrm{~h}$ & $72 \mathrm{~h}$ \\
\hline \multirow[t]{6}{*}{ Goldex } & 1 & $84 \mathrm{~b}$ & $74 \mathrm{~b}$ & $76 \mathrm{c}$ & $85 \mathrm{~b}$ & $80 \mathrm{~b}$ & $78 \mathrm{c}$ \\
\hline & 2 & $94 \mathrm{a}$ & $88 \mathrm{a}$ & $92 \mathrm{a}$ & $94 \mathrm{a}$ & $92 \mathrm{a}$ & $94 \mathrm{a}$ \\
\hline & 3 & 93 a & $90 \mathrm{a}$ & $92 \mathrm{a}$ & $92 a b$ & $88 a b$ & $90 \mathrm{ab}$ \\
\hline & 4 & $94 \mathrm{a}$ & $92 \mathrm{a}$ & $94 \mathrm{a}$ & $94 \mathrm{a}$ & $94 \mathrm{a}$ & $94 \mathrm{a}$ \\
\hline & 5 & $86 \mathrm{~b}$ & $76 \mathrm{~b}$ & $80 \mathrm{~b}$ & $90 \mathrm{ab}$ & $84 \mathrm{~b}$ & $88 \mathrm{~b}$ \\
\hline & CVs (\%) & 5,5 & 7,3 & 6,7 & 5,2 & 7,5 & 6,8 \\
\hline \multirow[t]{6}{*}{ Vereda } & 1 & $94 \mathrm{a}$ & $90 a$ & $92 \mathrm{a}$ & $92 \mathrm{a}$ & $88 a$ & $90 \mathrm{a}$ \\
\hline & 2 & $82 \mathrm{~b}$ & $80 \mathrm{~b}$ & $80 \mathrm{~b}$ & $84 \mathrm{~b}$ & $80 \mathrm{~b}$ & $82 \mathrm{~b}$ \\
\hline & 3 & 94 a & $84 \mathrm{~b}$ & $90 \mathrm{a}$ & $88 a b$ & $86 a$ & $84 \mathrm{~b}$ \\
\hline & 4 & $84 \mathrm{~b}$ & $82 \mathrm{~b}$ & $84 \mathrm{~b}$ & $82 \mathrm{~b}$ & $80 \mathrm{~b}$ & $80 \mathrm{~b}$ \\
\hline & 5 & $80 \mathrm{~b}$ & $80 \mathrm{~b}$ & $76 \mathrm{c}$ & $83 \mathrm{~b}$ & $78 \mathrm{~b}$ & $74 \mathrm{c}$ \\
\hline & CV s (\%) & 7,2 & 7,8 & 6,9 & 6,8 & 5,8 & 7,0 \\
\hline
\end{tabular}

Médias seguidas pela mesma letra na coluna não diferem entre si, pelo teste de Tukey, $\mathrm{p} \leq 0,05$, para cada híbrido (means followed by the same letter in the column did not differ from each other, Tukey's test, $\mathrm{p} \leq 0,05$ ).

\& Marcos Filho (2003), com sementes de melão. Além de apresentar a vantagem de possibilitar respostas mais rápidas sobre a qualidade dos lotes do que o período de 192 horas de envelhecimento, a $42^{\circ} \mathrm{C}$, sugerido por Pesis \& Timothy (1983), também para sementes de melão.

Verifica-se, de forma geral, que a utilização do procedimento tradicional ou modificado do teste de envelhecimento acelerado possibilitou a obtenção de resultados semelhantes quanto à classificação dos lotes de sementes em relação ao seu potencial fisiológico. Porém, o procedimento utilizando solução salina apresenta uma vantagem em relação ao procedimento tradicional: o uso de soluções salinas em substituição à água inibe sensivelmente o crescimento e desenvolvimento de fungos, facilitando o manuseio e eliminando sua possível interferência na avaliação do potencial fisiológico das sementes. Observações semelhantes foram feitas por Torres \& Marcos Filho (2003), com sementes de melão e Costa et al. (2008), com sementes de couve, couve-brócolis e repolho.

Nesse sentido, os resultados obtidos no presente trabalho indicaram que o teste de envelhecimento acelerado (procedimento tradicional e com solução salina saturada), utilizando o período de 72 horas, a $41^{\circ} \mathrm{C}$, mostra-se ade-
CARVALHO NM; NAKAGAWA J. 2000. Sementes: ciência, tecnologia e produção. 4 ed. Jaboticabal: Funep, 588p.

CNPq - Conselho Nacional de Desenvolvimento Científico e Tecnológico. 2007, 09 de outubro. Pesquisa financiada pelo $\mathrm{CNPq}$ garante qualidade na produção sustentável do melão. Disponível em http://www.cnpq.br/ saladeimprensa/noticias

CESM/RS - Comissão Estadual de Sementes e Mudas. 1998. Normas e padrões de sementes para o estado do Rio Grande do Sul. Porto Alegre: DPV, 155p.

COSTA CJ; TRZECIAK MB; VILLELA FA. 2008. Potencial fisiológico de sementes de brássicas com ênfase no teste de envelhecimento acelerado. Horticultura Brasileira 26: 144-148.

DELOUCHE JC; BASKIN CC. 1973. Accelerated aging techniques for predicting the relative storability of seed lots. Seed Science and Technology 1: 427-452.

DIAS DCFS; BHERING MC; TOKUHISA D; HILST PC. 2006. Teste de condutividade elétrica para avaliação do vigor de sementes de cebola. Revista Brasileira de Sementes 28: 154-162.

DIAS DCFS; VIEIRAAN; BHÉRING MC; 1998. Condutividade elétrica e lixiviação de potássio para avaliação do vigor de sementes de hortaliças: feijão-de-vagem e quiabo. Revista Brasileira de Sementes 20: 408-413.

FREITAS RA; NASCIMENTO WM. 2006. Teste de envelhecimento acelerado em sementes de lentilha. Revista Brasileira de Sementes 28: 59-63.

ABDO MTVN; PIMENTA RS; PANOBIANCO M; VIEIRA RD. 2005. Testes de vigor para avaliação de sementes de pepino. Revista Brasileira de Sementes 27: 195-198.

ASSOCIATION OF OFFICIAL SEED ANALYSTS. 1983. Seed vigor testing handbook. East Lansing: AOSA. 88p. (Contribution 32).

ÁVILA PFV; VILLELA FA; ÁVILA MS. 2006. Teste de envelhecimento acelerado para avaliação do potencial fisiológico de sementes de rabanete. Revista Brasileira de Sementes 28: 52-58.

BEWLEY JD; BLACK M. 1994. Seeds: physiology of development and germination. 2. ed. New York: Plenum Press. 445p.

BHERING MC; BARROS DI; DIAS DCFS; NUNES HV. 2001. Avaliação do vigor de sementes de melancia pelos testes de envelhecimento acelerado e deterioração controlada. Informativo ABRATES 11: 204.

BHÉRING MC; DIAS DCFS; GOMES JM; BARROS DI. 2000. Métodos para avaliação do vigor de sementes de pepino. Revista Brasileira de Sementes 22: 171-175.

BRASIL. Ministério da Agricultura e Reforma Agrária. 1992. Regras para análise de sementes. Brasília: SNDA/DNDV/CLAV. 365p.

CANO-RÍOS P; RAMÍREZ-ROSALES G; ORTEGÓN-PÉREZ J; ESPARZAMARTÍNEZ JH; RODRÍGUEZ-HERRERA S. 2000. Análisis dialélico para vigor de semilla en melón. Agrociencia 34: 337-342.
JIANHUA Z; McDONALD MD. 1996. The saturated salt accelerated aging test for small-seeded crops. Seed Science and Technology 25: 123-131.

MARCOS FILHO J. 1998. O valor dos testes de vigor. Seed News, 6: p.32.

MARCOS FILHO J. 1999a. Testes de vigor: importância e utilização. In: KRZYZANOWSKI FC; VIEIRA RD; FRANÇA NETO JB (eds). Vigor de sementes: conceitos e testes. Londrina: ABRATES. p.1.1-1.21.

MARCOS FILHO J. 1999b. Teste de envelhecimento acelerado. In: KRZYZANOWSKI FC; VIEIRA RD; FRANÇA NETO JB (eds). Vigor de sementes: conceitos e testes. Londrina: ABRATES. p.3.1-3.24.

McDONALD MB. 1995. Standardization of seed vigour tests. In: CONGRESS OF THE INTERNATIONAL SEED TESTING ASSOCIATION, 24. Proceedings... Copenhagen: ISTA. P.88-97.

NAKAGAWA J. 1994. Testes de vigor baseados na avaliação das plântulas. In: VIEIRA RD; CARVALHO NM (eds). Testes de vigor em sementes. Jaboticabal: Funep. p.49-86.

NASCIMENTO WM; FREITAS RA; GOMES EML; SOARES AS. 2007. Metodologia para o teste de envelhecimento acelerado em sementes de ervilha. Horticultura Brasileira 25: 205-209. 
NOVEMBRE ADLC; DIAS DCFS; CHAMMA HMCP; MARCOS FILHO J. 1995. Estudo da metodologia dos testes de envelhecimento acelerado e de condutividade elétrica para sementes de tomate. Informativo ABRATES 3: 140.

OLIVEIRA SRS; NOVEMBRE ADLC. 2005. Teste de condutividade elétrica para as sementes de pimentão. Revista Brasileira de Sementes 27: 31-36.

PESIS E; TIMOTHY JN. 1983. Viability, vigor, and electrolytic leakage of muskmelon seeds subjected to accelerated aging. Hortscience 18: 242-244.

POWELL AA. 1986. Cell membranes and seed leachate conductivity in relation to the quality of seed for sowing. Journal of Seed Technology 10: 81-100.
RODO AB; PERLEBERG CS; TORRES SB GENTIL DFO; TESSARIOLI NETO J. 2001. Qualidade fisiológica e tamanho de sementes de cenoura. Scientia Agrícola 58: 201-204.

SÁ ME. 1999. Condutividade elétrica em sementes de tomate (Lycopersicon lycopersicum L.). Scientia Agrícola 56: 1319.

TORRES SB; CASEIRO RF; RODO AB; MARCOS FILHO J. 1998. Testes de vigor em sementes de maxixe (Cucumis anguria L.) com ênfase ao teste de condutividade elétrica. Revista Brasileira de Sementes 20: 480-483.

TORRES SB; MARCOS FILHO J. 2003. Accelerated aging melon seeds. Scientia Agrícola 60: 77-82.
TORRES SB; MARCOS FILHO J. 2005. Physiological potential evaluation in melon seeds (Cucumis melo L.). Seed Science and Techonology 33: 341-350.

VIEIRA RD; DUTRA, AS. 2006. Condutividade elétrica em sementes de abóbora, híbrido Bárbara. Horticultura Brasileira 24: 305308.

VIEIRA RD; KRZYZANOWSKI FC. 1999. Teste de condutividade elétrica. In: KRZYZANOWSKI FC; VIEIRA RD; FRANÇA NETO JB (eds). Vigor de sementes: conceitos e testes. Londrina: ABRATES. p.4.1-4.26. 